\title{
Embeddings of maximal tori in orthogonal groups
}

\author{
Eva Bayer-Fluckiger
}

Abstract : We give necessary and sufficient conditions for an orthogonal group defined over a global field of characteristic $\neq 2$ to contain a maximal torus of a given type.

\section{Introduction}

Embeddings of maximal tori in orthogonal groups have been studied in several papers, and occur in various arithmetic questions (see for instance $[\mathrm{BCM}],[\mathrm{F}],[\mathrm{G}],[\mathrm{GR}],[\mathrm{L}],[\mathrm{PR}]$ and the references therein). The aim of this paper is to give necessary and sufficient conditions for an orthogonal group defined over a global field of characteristic $\neq 2$ to contain a maximal torus of a given type (see Theorem 3.2.1). As we will see, this gives rise to generalizations of some of the results of $[\mathrm{F}],[\mathrm{L}]$ and $[\mathrm{PR}]$ (see Theorem 3.1.1 and Corollary 3.1.2).

The case of tori of type $C M$ (that is, tori associated to CM étale algebras, see 1.2. and $\S 4)$ is of special interest in some of the applications, and will be used here to illustrate the results of the paper. The following is proved in $\S 4$ :

Theorem. Let $(E, \sigma)$ be a $\mathbf{Q}$-étale algebra with involution of type $C M$ of rank $2 n$, and let $q$ be a quadratic space over $\mathbf{Q}$ with $\operatorname{dim}(q)=\operatorname{rank}(E)$. Then the orthogonal group $O(q)$ contains a maximal torus of type $(E, \sigma)$ if and only if $\operatorname{disc}(q)=\operatorname{disc}(E) \in k^{*} / k^{* 2}$, the hyperbolicity condition holds (cf. 2.4), and the signature of $q$ is even.

In particular, a torus of type CM can be embedded as a maximal torus of an orthogonal group if and only if such an embedding exists everywhere locally.

\section{$\S 1$. Definitions, notation and basic facts}

Let $k$ be a field of characteristic $\neq 2$.

\subsection{Quadratic spaces}

A quadratic space is a symmetric bilinear form of non-zero determinant $q: V \times V \rightarrow k$, where $V$ is a finite dimensional $k$-vector space. We denote by $\operatorname{dim}(q)$ its $\operatorname{dimension}($ that is, the dimension of the underlying vector space $V)$, and by $O(q)$ its orthogonal group. The determinant of $q$ is denoted by $\operatorname{det}(q)$; it is an element of $k^{\times} / k^{\times 2}$. Let $m=\operatorname{dim}(q)$. Then the discriminant of $q$ is by definition $\operatorname{disc}(q)=(-1)^{\frac{m(m-1)}{2}} \operatorname{det}(q)$. Let us denote by $\operatorname{Br}(k)$ the Brauer group of $k$, considered as an additive abelian group, and let $\mathrm{Br}_{2}(k)$ be the subgroup of elements of order $\leq 2$ of $\operatorname{Br}(k)$. Any quadratic space can be diagonalized, in other words there exist $a_{1}, \ldots, a_{m} \in k^{\times}$such that $q \simeq<a_{1}, \ldots, a_{m}>$. The Hasse invariant of $q$ is by definition $\Sigma_{i<j}\left(a_{i}, a_{j}\right) \in \operatorname{Br}_{2}(k)$, where $\left(a_{i}, a_{j}\right)$ is the class of the quaternion algebra over $k$ determined by $a_{i}, a_{j}$, and is denoted by $w(q)$. If $q$ and $q^{\prime}$ are two quadratic spaces over $k$, then we denote by $q \oplus q^{\prime}$ their orthogonal sum. We have $w\left(q \oplus q^{\prime}\right)=w(q)+w\left(q^{\prime}\right)+\left(\operatorname{det}(q), \operatorname{det}\left(q^{\prime}\right)\right)$ (see for instance [Sch, 2.12.6]). 
If $q: V \times V \rightarrow k$ is a quadratic space, let us denote by $\tau_{q}: \operatorname{End}(V) \rightarrow \operatorname{End}(V)$ the adjoint involution of $q$; recall that we have $q(f(x), y)=q\left(x, \tau_{q}(f)(y)\right)$ for all $f \in \operatorname{End}(V)$ and all $x, y \in V$.

\subsection{Maximal tori and étale algebras with involution}

Recall that an étale algebra is a product of separable field extensions of finite degree of $k$. If $E$ is an étale algebra and $\sigma: E \rightarrow E$ is a $k$-linear involution, we denote by $E^{\sigma}$ the subalgebra of $E$ fixed by $\sigma$. The unitary group $U(E, \sigma)$ is by definition the linear algebraic group over $k$ defined by $U(E, \sigma)(A)=\left\{x \in E \otimes_{k} A \mid x \sigma(x)=1\right\}$ for any commutative $k$-algebra $A$. The following result is well-known (see for instance [BCM, 3.3], or [PR, 2.3]).

Proposition 1.2.1. Let $q: V \times V \rightarrow k$ be a quadratic space with $\operatorname{dim}(q)=2 n$. Then we have

(i) Let $T \subset O(q)$ be a maximal $k$-torus. Then there is a unique étale algebra $E \subset \operatorname{End}(V)$ stable by $\tau_{q}$ such that $T=U\left(E, \tau_{q}\right)$. Moreover, $E$ has rank $2 n$ and $E^{\tau_{q}}$ has rank $n$.

(ii) Conversely, for any étale algebra $E \subset \operatorname{End}(V)$ stable under $\tau_{q}$ and satisfying the rank conditions above, the unitary group $U\left(E, \tau_{q}\right)$ is a maximal $k$-torus of $O(q)$.

If $q: V \times V \rightarrow k$ is a quadratic space and $E$ an étale algebra with involution $\sigma: E \rightarrow E$, we say that a maximal torus $T$ of $O(q)$ is of type $(E, \sigma)$ if the conditions of Proposition 1.2.1 hold for some étale algebra $E^{\prime} \subset \operatorname{End}(V)$ such that the algebras with involution $(E, \sigma)$ and $\left(E^{\prime}, \tau_{q} \mid E^{\prime}\right)$ are isomorphic, in particular $T \simeq U(E, \sigma)$.

\subsection{Realizable pairs}

If $\left(A_{1}, \tau_{1}\right)$ and $\left(A_{2}, \tau_{2}\right)$ are two $k$-algebras with involution. An embedding of $\left(A_{1}, \tau_{1}\right)$ in $\left(A_{2}, \tau_{2}\right)$ is by definition an injective homomorphism of algebras $A_{1} \rightarrow A_{2}$ that commutes with the involutions.

For any étale algebra with involution $(E, \sigma)$ and any $\alpha \in E^{\sigma}$, let $q_{\alpha}: E \times E \rightarrow k$ be the symmetric bilinear form $q_{\alpha}(x, y)=\operatorname{Tr}_{E / k}(\alpha x \sigma(y))$. The following proposition is well-known

Proposition 1.3.1. Let $(E, \sigma)$ be an étale algebra with involution of rank $2 n$, and assume that the rank of $E^{\sigma}$ is $n$. Let $q: V \times V \rightarrow k$ be a $2 n$-dimensional quadratic space. Then the following are equivalent :

(i) The orthogonal group $O(q)$ contains a maximal torus of type $(E, \sigma)$.

(ii) The algebra with involution $(E, \sigma)$ can be embedded in the algebra with involution (End $\left.(V), \tau_{q}\right)$.

(iii) There exists $\alpha \in E^{\sigma}$ such that $q \simeq q_{\alpha}$.

Proof. The equivalence of (i) and (ii) follows from Proposition 1.2.1. For the equivalence of (ii) and (iii), see for instance [PR, 7.1].

We say that the pair $(E, q)$ is realizable if the equivalent conditions of Proposition 1.2.1 hold. Recall that the discriminant of the étale algebra $E$ is by definition the determinant 
of the quadratic space $E \times E \rightarrow k$ given by $(x, y) \mapsto \operatorname{Tr}_{E / k}(x y)$. It is denoted by $\operatorname{disc}(E)$. The following lemma is well-known, see for instance [F, 3.3.1] :

Lemma 1.3.2. If $(E, q)$ is realizable, then $\operatorname{disc}(q)=\operatorname{disc}(E) \in k^{\times} / k^{\times 2}$.

Proof. As $q$ is realizable, we have $q=q_{\alpha}$ for some $\alpha \in E^{\sigma}$. Let $Q=q_{1}: E \times E \rightarrow k$ and $Q^{\prime}: E \times E \rightarrow k$ be the quadratic spaces defined by $Q(x, y)=\operatorname{Tr}_{E / k}(x \sigma(y))$ and $Q^{\prime}(x, y)=$ $\operatorname{Tr}_{E / k}(x y)$. We have $\operatorname{disc}(q)=\mathrm{N}_{E / k}(\alpha) \operatorname{disc}(Q)$. As $\alpha \in E^{\sigma}$, we have $\mathrm{N}_{E / k}(\alpha) \in k^{2}$, hence $\operatorname{disc}(q)=\operatorname{disc}(Q)$. Writing $E=E^{\sigma}(\sqrt{\theta})$ for some $\theta \in E^{\sigma}$, a straightforward computation shows that $\operatorname{det}\left(Q^{\prime}\right)=(-1)^{n} \operatorname{det}(Q)$. As $\operatorname{disc}(E)=\operatorname{det}\left(Q^{\prime}\right)$ and $\operatorname{disc}(Q)=(-1)^{n} \operatorname{det}(Q)$ by definition, the result follows.

\section{$\S$ 2. Local conditions}

Suppose that $k$ is a global field, and let us denote by $\Sigma_{k}$ the set of places of $k$. We keep the notation of $\S 1$. Let $n \in \mathbf{N}$, and let $(E, \sigma)$ be an étale algebra with involution of rank $2 n$. Suppose that $E=K_{1} \times \ldots \times K_{r}$, where $K_{1}, \ldots, K_{r}$ are separable extensions of $k$, and that the $K_{i}$ 's are all stable by $\sigma$. Let $I=\{1, \ldots, r\}$, and for all $i \in I$, let us denote by $F_{i}$ the fixed field of $\sigma$ in $K_{i}$. Suppose that $K_{i}$ is a quadratic extension of $F_{i}$ for all $i \in I$. Note that $E^{\sigma}=F_{1} \times \ldots \times F_{r}$, and that $\operatorname{rank}\left(E^{\sigma}\right)=n$. Let $\Sigma_{k}^{\text {split }}(E)$ be the set of $v \in \Sigma_{k}$ such that all the places of $E^{\sigma}$ above $v$ split in $E$.

We start by giving some local conditions for the embedding question of the previous section.

\subsection{Split places}

Recall that a quadratic space $(V, q)$ is hyperbolic if there exists a subspace $W$ of $V$ such that $\operatorname{dim}(V)=2 \operatorname{dim}(W)$, and $q(x, y)=0$ for all $x, y \in W$. It is well-known that a hyperbolic space is uniquely determined up to isomorphism by its dimension. Let us denote by $h_{2 n}$ the hyperbolic space of dimension $2 n$.

Lemma 2.1.1. Let $v \in \Sigma_{k}^{\mathrm{split}}(E)$, and let $q$ be a $2 n$-dimensional quadratic space over $k_{v}$. Then $(E, q)$ is realizable over $k_{v}$ if and only if $q$ is hyperbolic.

Proof. As $v \in \Sigma_{k}^{\text {split }}(E)$, over $k_{v}$ we have an isomorphism $E \simeq E_{1} \times E_{2}$ where $E_{1}$ and $E_{2}$ are isomorphic étale $k_{v}$-algebras, and $\sigma\left(E_{1}\right)=E_{2}$. Let us show that $q_{\alpha}$ is hyperbolic for any $\alpha \in\left(E_{v}^{\sigma}\right)^{\times}$. Set $W=E_{1} \times\{0\}$. Then $x \sigma(y)=0$ for all $x, y \in W$, hence the restriction of $q_{\alpha}$ to $W$ is identically zero. Since $\operatorname{dim}_{k}(W)=\frac{1}{2} \operatorname{dim}_{k}(E)$, this proves that $q_{\alpha}$ is hyperbolic, hence $q_{\alpha} \simeq h_{2 n}$. Therefore $\left(E, h_{2 n}\right)$ is realizable over $k_{v}$. Conversely, if $(E, q)$ is realizable over $k_{v}$, we have $q \simeq q_{\alpha}$ for some $\alpha \in\left(E_{v}^{\sigma}\right)^{\times}$, hence by the previous argument $q \simeq h_{2 n}$.

\subsection{Non-split places}

Recall that if $v \in \Sigma_{k}$ is a finite place or a real place, then $\operatorname{Br}_{2}\left(k_{v}\right)$ is a cyclic group of order 2. We will identify it to $\{0,1\}$. The following results will be used several times in the sequel. 
Proposition 2.2.1. Let $v$ be a place of $k$ such that $v \notin \Sigma_{k}^{\mathrm{split}}(E)$. Let $\epsilon \in\{0,1\}$. Then there exists $\alpha \in\left(E_{v}^{\sigma}\right)^{\times}$such that $w\left(q_{\alpha}\right)=\epsilon$.

Proof. Recall that $q_{1}: E_{v} \times E_{v} \rightarrow k_{v}$ is defined by $q_{1}(x, y)=\operatorname{Tr}_{E_{v} / k_{v}}(x \sigma(y))$. If $w\left(q_{1}\right)=\epsilon$, we can take $\alpha=1$. Suppose that $w(q) \neq \epsilon$. As $v \notin \Sigma_{k}^{\text {split }}(E)$, we have $E_{v}=E^{\prime} \times K$, where $K$ is a field extension of $k_{v}$ stable by $\sigma$. Set $F=K^{\sigma}$. Then $K$ is a quadratic extension of $F$. Let $\beta \in F^{\times}$such that $\beta \notin \mathrm{N}_{K / F}\left(K^{\times}\right)$. Let us denote by $q_{1}^{\prime}$ the restriction of $q_{1}$ to $K$. Then we have $w\left(q_{\beta}\right) \neq w\left(q_{1}^{\prime}\right)$; this follows from [M, 2.7] if $v$ is a finite place, and it is clear if $v$ is an infinite place. Let $\alpha=(\beta, 1) \in E_{v}^{\sigma}$. Then $w\left(q_{\alpha}\right) \neq w\left(q_{1}\right)$, hence $w\left(q_{\alpha}\right)=\epsilon$.

Lemma 2.2.2. Suppose that there exists a real place $u$ of $k$ such that we have $u \notin \Sigma_{k}^{\text {split }}\left(K_{i}\right)$ for all $i \in I$. Then there exists a finite place $v$ of $k$ such that for all $i \in I$, we have $v \notin \Sigma_{k}^{\mathrm{split}}\left(K_{i}\right)$.

Proof. Let $L$ be a Galois extension of $k$ containing the fields $K_{i}$ for all $i \in I$. Let $G=\operatorname{Gal}(L / k)$. Let us denote by $c$ the conjugacy class of the complex conjugation in $G$ corresponding to an extension of the place $u$ to $L$. By the Chebotarev density theorem, there exists a finite place $v$ of $k$ such that the conjugacy class of the Frobenius automorphism at $v$ is equal to $c$. Let $v$ be such a place. Then all the places of $F_{i}$ above $v$ are inert in $K_{i}$. Therefore we have $v \notin \Sigma_{k}^{\text {split }}\left(K_{i}\right)$ for all $i \in I$, and the statement is proved.

\subsection{Real places}

Let $v$ be a real place of $k$. It is well-known that any quadratic space $q$ over $k_{v}$ is isomorphic to $X_{1}^{2}+\ldots+X_{r}^{2}-X_{r+1}^{2}-\ldots-X_{r+s}^{2}$ for some non-negative integers $r$ and $s$. These are uniquely determined by $q$, and we have $r+s=\operatorname{dim}(q)$. The couple $(r, s)$ is called the signature of $q$ at $v$. We say that the signature of $q$ at $v$ is even if $r \equiv s \equiv 0(\bmod 2)$, and we say that the signatures of $q$ are even if the signature of $q$ at $v$ is even for all real places $v$ of $k$.

We say that a place $w$ of $E^{\sigma}$ above $v$ is ramified in $E$ if $w$ is a real place that extends to a complex place of $E$. Let $\rho_{v}$ be the number of places of $E^{\sigma}$ above $v$ which are not ramified in $E$. The following lemma is well-known

Lemma 2.3.1. Let $\alpha \in\left(E^{\sigma}\right)^{\times}$. Then the signature of $q_{\alpha}$ is equal to $\left(2 r_{\alpha}+\rho_{v}, 2 s_{\alpha}+\rho_{v}\right)$ where $r_{\alpha}$ is the number of places of $E^{\sigma}$ above $v$ that ramify in $E$ at which $\alpha$ is positive, and $s_{\alpha}$ is the number places of $E^{\sigma}$ that ramify in $E$ at which $\alpha$ is negative.

Proof. This is immediate.

Proposition 2.3.2. Let $q$ be a $2 n$-dimensional quadratic space over $k_{v}$. Then $(E, q)$ is realizable if and only if the signature of $q$ is of the shape $\left(2 r^{\prime}+\rho_{v}, 2 s^{\prime}+\rho_{v}\right)$ for some non-negative integers $r^{\prime}, s^{\prime}$.

Proof. If $(E, q)$ is realizable, then lemma 2.3.1. shows that the signature of $q$ has the required shape. Conversely, suppose that the signature of $q$ is equal to $\left(2 r^{\prime}+\rho_{v}, 2 s^{\prime}+\rho_{v}\right)$ for some $r^{\prime}, s^{\prime} \in \mathbf{N}$. Let $\alpha \in\left(E^{\sigma}\right)^{\times}$be such that $\alpha$ is positive at $r^{\prime}$ places of $E^{\sigma}$ above $v$ and 
negative at $s^{\prime}$ places. Then by lemma 2.3.1, the signature of $q_{\alpha}$ is equal to $\left(2 r^{\prime}+\rho_{v}, 2 s^{\prime}+\rho_{v}\right)$. This implies that $q \simeq q_{\alpha}$, hence $(E, q)$ is realizable.

\subsection{Combining local criteria}

If $q$ is a $2 n$-dimensional quadratic space over $k$, we say that the signature condition holds for $E$ and $q$ if for every real place $v$ of $k$, the signature of $q$ at $v$ is of the shape $\left(2 r^{\prime}+\rho_{v}, 2 s^{\prime}+\rho_{v}\right)$ for some non-negative integers $r^{\prime}, s^{\prime}$. For all $a \in \operatorname{Br}(k)$ and all $v \in \Sigma_{k}$, let us denote by $a_{v}$ the image of $a$ in $\operatorname{Br}\left(k_{v}\right)$. Recall that $h_{2 n}$ is the $2 n$-dimensional hyperbolic space. We say that the hyperbolicity condition holds for $E$ and $q$ if for all $v \in \Sigma_{k}^{\text {split }}(E)$, we have $w(q)_{v}=w\left(h_{2 n}\right)_{v}$.

Proposition 2.4.1. Let $q$ be a $2 n$-dimensional quadratic space over $k$. Then $(E, q) i s$ realizable over all the completions of $k$ if and only if $\operatorname{disc}(q)=\operatorname{disc}(E) \in k^{*} / k^{* 2}$, and if the hyperbolicity condition and the signature condition hold for $q$ and $E$.

Proof. Suppose that $\operatorname{disc}(q)=\operatorname{disc}(E) \in k^{*} / k^{* 2}$, and that the hyperbolicity condition and the signature condition hold. Let us prove that $(E, q)$ is realizable over $k_{v}$ for all $v \in \Sigma_{k}$. Suppose first that $v$ is an infinite place. If $v$ is complex, then there is nothing to prove. If $v$ is a real place, then by Proposition 2.3.2 the signature condition implies that $(E, q)$ is realizable over $k_{v}$. Suppose now that $v$ is a finite place. If $v \in \Sigma_{k}^{\text {split }}(E)$, then the equality $\operatorname{disc}(q)=\operatorname{disc}(E) \in k^{*} / k^{* 2}$ and the hyperbolicity condition imply that the discriminants and the Hasse invariants of $q$ and of $h_{2 m}$ coincide over $k_{v}$. Therefore $q \simeq h_{2 n}$ over $k_{v}$, and by Lemma 2.1.1 this implies that $(E, q)$ is realizable over $k_{v}$. Suppose that $v \notin \Sigma_{k}^{\text {split }}(E)$. By Proposition 2.2.1, there exists $\alpha \in\left(E_{v}^{\sigma}\right)^{\times}$such that $w\left(q_{\alpha}\right)=w(q)_{v}$. By Lemma 1.3.2, we have $\operatorname{disc}\left(q_{\alpha}\right)=\operatorname{disc}(E)$. As by hypothesis $\operatorname{disc}(q)=\operatorname{disc}(E) \in k^{*} / k^{* 2}$, the discriminants of $q$ and $q_{\alpha}$ are equal in $k_{v}^{\times} / k_{v}^{\times 2}$. Therefore $q$ and $q_{\alpha}$ are isomorphic over $k_{v}$, and this implies that $(E, q)$ is realizable over $k_{v}$. The converse follows immediately from Lemmas 1.3.2 and 2.1.1, and from Proposition 2.3.2.

\section{$\S 3$. Embedding criteria and Hasse principle}

We keep the notation of the previous sections. In particular, $k$ is a global field of characteristic $\neq 2$, and $(E, \sigma)$ is étale algebra with involution of rank $2 n$ such that $E=$ $K_{1} \times \ldots \times K_{r}$, where $K_{1}, \ldots, K_{r}$ are separable extensions of $k$, the $K_{i}$ 's are all stable by $\sigma$, and $F_{i}$ is the fixed field of $\sigma$ in $K_{i}$ for all $i \in I=\{1, \ldots, r\}$.

Recall that $\Sigma_{k}$ is the set of places of $k$, and that $\Sigma_{k}^{\text {split }}\left(K_{i}\right)$ is the set of $v \in \Sigma_{k}$ such that all the places of $F_{i}$ above $v$ split in $K_{i}$. For all $i \neq j$, set $\Sigma_{i, j}=\Sigma_{k}^{\text {split }}\left(K_{i}\right) \cup \Sigma_{k}^{\text {split }}\left(K_{j}\right)$.

\subsection{Sufficient conditions and some notation}

One of the results of this section is the following local-global principle

Theorem 3.1.1. Suppose that there exists $i_{0} \in I$ such that for all $i \in I$, we have $\Sigma_{i_{0}, i} \neq$ $\Sigma_{k}$. Let $q$ be a $2 n$-dimensional quadratic space. Then a torus of type $(E, \sigma)$ can be embedded 
in the orthogonal group $O(q)$ if and only if such an embedding exists over all the completions of $k$.

Note that this implies [PR, 7.3] and [L, 2.20]. As we will see, Theorem 3.1.1 is a consequence of Theorem 3.2.1 below. We also get the following corollary, which provides an embedding criterion in terms of invariants of the étale algebra and the quadratic space.

Corollary 3.1.2. Suppose that there exists $i_{0} \in I$ such that for all $i \in I$, we have $\Sigma_{i_{0}, i} \neq \Sigma_{k}$. Then $O(q)$ contains a maximal torus of type $(E, \sigma)$ if and only if $\operatorname{disc}(q)=$ $\operatorname{disc}(E) \in k^{*} / k^{* 2}$ and the signature and hyperbolicity conditions hold.

Proof. This follows from Proposition 2.4.1 and Theorem 3.1.1.

The following results will be needed in the proof of Theorem 3.1.1.

Proposition 3.1.3. Suppose that $(E, q)$ is realizable over all the completions of $k$. Then for all places $v$ of $k$ and $i \in I$, there exist quadratic spaces $q_{i}^{v}$ over $k_{v}$ such that

(i) for all $i \in I$ and every place $v$ of $k$, the pair $\left(K_{i}^{v}, q_{i}^{v}\right)$ is realizable;

(ii) for every place $v$ of $k$, we have $q \simeq q_{1}^{v} \oplus \ldots \oplus q_{r}^{v}$;

(iii) for all $i \in I$, we have $w\left(q_{i}^{v}\right)=0$ for almost all $v \in \Sigma_{k}$.

Proposition 3.1.3 is an immediate consequence of Proposition 3.1.4 below, in which condition (iii) is replaced by the more precise condition (iii'). Let us start by introducing some notation, that will be needed several times in the sequel. For all $i \in I$, let $n_{i}=\left[K_{i}: k\right]$, let $d_{i}=(-1)^{n_{i}} \operatorname{disc}\left(K_{i}\right)$, and set $D=\Sigma_{i<j}\left(d_{i}, d_{j}\right) \in \operatorname{Br}_{2}(k)$. Recall that for all $a \in \operatorname{Br}(k)$ and all $v \in \Sigma_{k}$, we denote by $a_{v}$ the image of $a$ in $\operatorname{Br}\left(k_{v}\right)$. Let $T$ be the set of places $v$ of $k$ such that $D_{v} \neq 0$, and let $S$ be the set of places of $k$ at which the Hasse invariant of $q$ is not equal to the Hasse invariant of the hyperbolic form of dimension equal to $\operatorname{dim}(q)$. Let $\Sigma_{2}$ be the set of dyadic places and $\Sigma_{\infty}$ the set of infinite places of $k$, and set $\Sigma=S \cup T \cup \Sigma_{2} \cup \Sigma_{\infty}$. Note that $\Sigma$ is a finite set.

Proposition 3.1.4. Suppose that $(E, q)$ is realizable over all the completions of $k$. Then for all places $v$ of $k$ and $i \in I$, there exist quadratic spaces $q_{i}^{v}$ over $k_{v}$ such that

(i) for all $i \in I$ and every place $v$ of $k$, the pair $\left(K_{i}^{v}, q_{i}^{v}\right)$ is realizable;

(ii) for every place $v$ of $k$, we have $q \simeq q_{1}^{v} \oplus \ldots \oplus q_{r}^{v}$;

(iii') for all $i \in I$, we have $w\left(q_{i}^{v}\right)=0$ if $v \notin \Sigma$.

Proof. Let $v$ be a place of $k$. By hypothesis, $(E, q)$ is realizable over $k_{v}$. Hence there exists $\alpha \in\left(E_{v}^{\sigma}\right)^{\times}$such that $q \simeq q_{\alpha}$ over $k_{v}$, and we have $\alpha=\left(\alpha_{1}, \ldots, \alpha_{r}\right)$ with $\alpha_{i} \in\left(F_{i}^{v}\right)^{\times}$. Then the quadratic spaces $q_{i}^{v}=q_{\alpha_{i}}$ fulfill conditions (i) and (ii). Let us show that we can change the $q_{i}^{v}$ so that condition (iii') holds as well.

Let $v \in \Sigma_{k}$ be such that $v \notin \Sigma$, and suppose that there exists $i \in I$ with $w\left(q_{i}^{v}\right)=1$. Let us show that there exist quadratic spaces $\tilde{q}_{j}^{v}$ for all $j \in I$ such that $w\left(\tilde{q}_{j}^{v}\right)=0$ if $w\left(q_{j}^{v}\right)=0$, and $w\left(\tilde{q}_{i}^{v}\right)=0$. As $v \notin S \cup \Sigma_{2}$, we have $w(q)_{v}=0$. Note that $w(q)_{v}=$ $w\left(q_{1}^{v}\right)+\ldots+w\left(q_{r}^{v}\right)+D_{v}$, and as $v \notin T$, we have $D_{v}=0$. Therefore there exists $m \in I$ with $m \neq i$ such that $w\left(q_{m}^{v}\right)=1$. As $v$ is not dyadic, this implies that $q_{i}^{v}$ and $q_{m}^{v}$ are not 
hyperbolic, hence by Lemma 2.1.1 we have $v \notin \Sigma_{i, m}$. As $v \notin \Sigma_{k}^{\text {split }}\left(K_{i}\right)$, by Proposition 2.2.1 there exists $\beta_{i} \in F_{i}^{v}$ such that $w\left(q_{\beta_{i}}\right)=0$. Similarly, as $v \notin \Sigma_{k}^{\text {split }}\left(K_{m}\right)$, there exists $\beta_{m} \in F_{m}^{v}$ such that $w\left(q_{\beta_{m}}\right)=0$. Let $\tilde{q}_{i}^{v}=q_{\beta_{i}}$ and $\tilde{q}_{m}^{v}=q_{\beta_{m}}$, and set $\tilde{q}_{j}^{v}=q_{j}^{v}$ for $j \neq i, m$. We have $w\left(\tilde{q}_{j}^{v}\right)=0$ if $w\left(q_{j}^{v}\right)=0$, and $w\left(\tilde{q}_{i}^{v}\right)=0$. By Lemma 1.3 .2 we have $\operatorname{det}\left(\tilde{q}_{j}^{v}\right)=\operatorname{det}\left(q_{j}^{v}\right)$ for all $j \in I$. Moreover, as $w\left(\tilde{q}_{i}^{v}\right)=0$ and $w\left(\tilde{q}_{m}^{v}\right)=0$, we have $w\left(\tilde{q}_{1}^{v} \oplus \ldots \oplus \tilde{q}_{r}^{v}\right)=w\left(q_{1}^{v} \oplus \ldots \oplus q_{r}^{v}\right)$, implying that $\tilde{q}_{1}^{v} \oplus \ldots \oplus \tilde{q}_{r}^{v} \simeq q_{1}^{v} \oplus \ldots \oplus q_{r}^{v}$. Therefore condition (ii) holds. The pairs $\left(K_{j}^{v}, \tilde{q}_{j}^{v}\right)$ are realizable for all $j \in I$, hence condition (i) holds as well. Repeating this procedure for all $i \in I$ with $w\left(q_{i}^{v}\right)=1$ and for all $v \in \Sigma_{k}$ with $v \notin \Sigma$ leads to quadratic spaces over $k_{v}$ satisfying all three conditions. This concludes the proof of the proposition.

\subsection{A necessary and sufficient condition}

In order to state a necessary and sufficient condition for the embedding problem of tori in orthogonal groups (see Theorem 3.2.1 below), we need the following notation and definition

Notation. Let $\mathcal{C}(E, q)$ be the set of collections $\left(q_{i}^{v}\right)$ of quadratic spaces over $k_{v}$ satisfying conditions (i) - (iii) of Proposition 3.1.3. For $C=\left(q_{i}^{v}\right) \in \mathcal{C}(E, q)$ and $i \in I$, set

$$
S_{i}(C)=\left\{v \in \Sigma_{k}^{\prime} \mid w\left(q_{i}^{v}\right)=1\right\} .
$$

By condition (iii) $S_{i}(C)$ is a finite set, and we denote by $\left|S_{i}(C)\right|$ its cardinal.

Definition. We say that $C=\left(q_{i}^{v}\right) \in \mathcal{C}(E, q)$ is connected if for all $i \in I$ such that $\left|S_{i}(C)\right|$ is odd, there exist $j \in I$ with $j \neq i$ such that $\left|S_{j}(C)\right|$ is odd, and a chain $i=i_{1}, \ldots, i_{m}=j$ of elements of $I$ with $\Sigma_{i_{t}, i_{t+1}} \neq \Sigma_{k}$ for all $t=1, \ldots, m-1$. We say that $\mathcal{C}(E, q)$ is connected if it contains a connected element.

Theorem 3.2.1. Let $q$ be a $2 n$-dimensional quadratic space. Then :

(a) The orthogonal group $O(q)$ contains a torus of type $(E, \sigma)$ over all completions of $k$ if and only if $\mathcal{C}(E, q)$ is not empty.

(b) The orthogonal group $O(q)$ contains a torus of type $(E, \sigma)$ if and only if $\mathcal{C}(E, q)$ is connected.

Proof. (a) With the terminology of 1.3 , we have to show that $(E, q)$ is realizable over all completions of $k$ if and only if $\mathcal{C}(E, q)$ not empty. It is clear that if $\mathcal{C}(E, q)$ not empty, then $(E, q)$ is realizable over $k_{v}$ for all $v \in \Sigma_{k}$, and the converse follows from Proposition 3.1.3.

(b) We have to prove that $(E, q)$ is realizable over $k$ if and only if $\mathcal{C}(E, q)$ is connected. If $(E, q)$ is realizable, then there exist quadratic spaces $q_{1}, \ldots, q_{r}$ over $k$ such that $q \simeq$ $q_{1} \oplus \ldots \oplus q_{r}$ and that $\left(K_{i}, q_{i}\right)$ is realizable over $k$ for all $i \in I$. Set $q_{i}^{v}=q_{i} \otimes_{k} k_{v}$, and let $C=\left(q_{i}^{v}\right)$. Then $C \in \mathcal{C}(E, q)$, and $\left|S_{i}(C)\right|$ is even for all $i \in I$. Therefore $C$ is a connected element of $\mathcal{C}(E, q)$, hence $\mathcal{C}(E, q)$ is connected.

Conversely, suppose that $\mathcal{C}(E, q)$ is connected, and note that by part (a) this implies that $(E, q)$ is realizable over all the completions of $k$. Let us show that $(E, q)$ is realizable.

Step 1. If $r=1$, then $(E, q)$ is realizable. This can be deduced from [PR, 7.4] or [F, 1.1], but we give a (different) proof for the convenience of the reader. Let $v$ be a real place of 
$k$ and let $\left(r_{v}, s_{v}\right)$ be the signature of $q$ at $v$. As $(E, q)$ is realizable over $k_{v}$ by hypothesis, Proposition 2.3.2 implies that $\left(r_{v}, s_{v}\right)=\left(2 r_{v}^{\prime}+\rho_{v}, 2 s_{v}^{\prime}+\rho_{v}\right)$ for some $r_{v}^{\prime}, s_{v}^{\prime} \in \mathbf{N}$. Let $\alpha \in E^{\sigma}$ be such that $\alpha$ is positive at exactly $r_{v}^{\prime}$ real places of $E^{\sigma}$ that become complex in $E$. Then $\alpha$ is negative at exactly real $s_{v}^{\prime}$ places of $E^{\sigma}$ that become complex in $E$, hence by Lemma 2.3.1 the signature of $q_{\alpha}$ is $\left(r_{v}, s_{v}\right)$. Let $S_{k}$ be the set of places of $k$ at which $q_{\alpha}$ and $q$ are not isomorphic. Note that $S_{k}$ consists of finite places of $k$, and it is a finite set of even cardinality. If $v \in S_{k}$, then $v \notin \Sigma_{k}^{\text {split }}(E)$. Indeed, both $(E, q)$ and $\left(E, q_{\alpha}\right)$ are realizable over $k_{v}$ for all $v \in \Sigma_{k}$. If $v \in \Sigma_{k}^{\text {split }}(E)$, then by Lemma 2.1.1 this implies that $q$ and $q_{\alpha}$ are both hyperbolic over $k_{v}$, hence they are isomorphic over $k_{v}$, and therefore $v \notin S_{k}$. For all $v \in S_{k}$, let us choose a place $w$ of $E^{\sigma}$ that does not split in $E$ - this is possible because $v \notin \Sigma_{k}^{\text {split }}(E)$. Let us denote by $S_{E}$ the set of these places $w$. Then $S_{E}$ is in bijection with $S$, hence it is also a finite set of even cardinality. Let us write $E=E^{\sigma}(\sqrt{\theta})$ for some $\theta \in\left(E^{\sigma}\right)^{\times}$, and let us choose $\beta \in\left(E^{\sigma}\right)^{\times}$such that $(\beta, \theta)_{w}=-1$ if $w \in S_{E}$ and $(\beta, \theta)_{w}=1$ otherwise. This is possible as $S_{E}$ has even cardinality (see for instance [O'M, 71.19], or $[\mathrm{PR}, 6.5])$. Then by $[\mathrm{M}, 2.7]$, the Hasse invariant of $q_{\alpha \beta}$ is equal to the Hasse invariant of $q$. Since these two quadratic spaces have equal dimension, determinant and signatures, they are isomorphic by the Hasse-Minkowski theorem. Therefore $(E, q)$ is realizable.

Step 2. Let us show that $\mathcal{C}(E, q)$ contains $C=\left(q_{i}^{v}\right)$ such that

(iv) $\left|S_{i}(C)\right|$ is even for all $i \in I$.

Let $C=\left(q_{i}^{v}\right) \in \mathcal{C}(E, q)$ be a connected element. Recall that by hypothesis $C$ satisfies conditions (i) - (iii) of Proposition 3.1.3. Suppose that for some $i \in I$, the integer $\left|S_{i}(C)\right|$ is odd. Since $C$ is connected, there exist $j \in I$ with $j \neq i$ such that $\left|S_{j}(C)\right|$ is odd, and a chain $i=i_{1}, \ldots, i_{m}=j$ of elements of $I$ with $\Sigma_{i_{t}, i_{t+1}} \neq \Sigma_{k}$ for all $t=1, \ldots, m-1$. For all $t=1, \ldots, m-1$, let $v_{t} \notin \Sigma_{i_{t}, i_{t+1}}$ be a finite place (note that this is possible by Lemma 2.2.2). Let $\alpha_{1} \in\left(F_{1}^{v_{1}}\right)^{\times}$be such that $q_{i}^{v_{1}} \simeq q_{\alpha_{1}}$ over $k_{v_{1}}$. By Proposition 2.2.1, there exist $\alpha_{t} \in\left(F_{t}^{v_{t}}\right)^{\times}$such that $w\left(q_{\alpha_{t}}\right) \neq w\left(q_{\alpha_{t+1}}\right)$ for all $t=1, \ldots, m-1$. Set $\tilde{q}_{t}^{v_{t}}=q_{\alpha_{t}}$ for all $t=1, \ldots, m-1$, and let $\tilde{q}_{s}^{u}=q_{s}^{u}$ if $(u, s) \neq\left(v_{t}, t\right)$. Set $\tilde{C}=\left(\tilde{q}_{i}^{v}\right)$. Then $\tilde{C} \in \mathcal{C}(E, q)$. We have $\left|S_{i}(\tilde{C})\right| \equiv 0(\bmod 2),\left|S_{j}(\tilde{C})\right| \equiv 0(\bmod 2)$, and $\left|S_{s}(\tilde{C})\right| \equiv\left|S_{s}(C)\right|(\bmod 2)$ if $s \neq i, j$. Repeating this procedure we obtain a family of quadratic spaces safisfying conditions (i) (iv).

Step 3. End of proof. Let $C=\left(q_{i}^{v}\right) \in \mathcal{C}(E, q)$ satisfy conditions (i) - (iv); this is possible by Step 2. For all $i \in I$, there exists a quadratic space $q_{i}$ over $k$ such that $q_{i}^{v} \simeq q_{i}$ over $k_{v}$ for all places $v$ of $k$. This follows from [O'M, 72.1], which applies because of conditions (iii) and (iv), and the fact that by condition (i) and Lemma 1.3.2 we have $\operatorname{disc}\left(q_{i}^{v}\right)=d_{i}$ for all places $v$ of $k$. By condition (ii) we have $q \simeq q_{1} \oplus \ldots \oplus q_{r}$ over all the completions of $k$, hence by the Hasse-Minkowski theorem $q \simeq q_{1} \oplus \ldots \oplus q_{r}$ over $k$ as well. Note that by condition (i), the pair $\left(K_{i}, q_{i}\right)$ is realizable over all the completions of $k$. By Step 1, this implies that $\left(K_{i}, q_{i}\right)$ is realizable over $k$, hence $(E, q)$ is realizable as well. This concludes the proof of the theorem.

Note that the conditions (a) and (b) of Theorem 3.2.1 are not equivalent, in other words the local-global principle does not hold in general : this follows from the examples of Prasad and Rapinchuk, cf. [PR, 7.5]. 
In order to deduce Theorem 3.1.1 from Theorem 3.2.1, we need the following lemma

Lemma 3.2.2. Let $C=\left(q_{i}^{v}\right) \in \mathcal{C}(E, q)$. Then $\Sigma_{i \in I}\left|S_{i}(C)\right| \equiv 0(\bmod 2)$.

Proof. For all $v \in \Sigma_{k}$, set $S_{v}(C)=\left\{i \in I \mid w\left(q_{i}^{v}\right)=1\right\}$. We have

$$
\Sigma_{v \in \Sigma}\left|S_{v}(C)\right|=\Sigma_{i \in I}\left|S_{i}(C)\right|
$$

By property (ii), we have $\left|S_{v}(C)\right| \equiv w(q)_{v}+D_{v} \quad(\bmod 2)$ for all $v \in \Sigma_{k}$. Therefore $\Sigma_{v \in \Sigma_{k}}\left|S_{v}\right|(C) \mid \equiv \Sigma_{v \in \Sigma_{k}} w(q)_{v}+\Sigma_{v \in \Sigma_{k}} D_{v}(\bmod 2)$. As $w(q)$ and $D$ are elements of $\operatorname{Br}_{2}(k)$, we have

$$
\Sigma_{v \in \Sigma_{k}^{\prime}} w(q)_{v} \equiv 0(\bmod 2), \text { and } \Sigma_{v \in \Sigma_{k}^{\prime}} D_{v} \equiv 0(\bmod 2) \text {. }
$$

This implies that $\Sigma_{v \in \Sigma}\left|S_{v}(C)\right| \equiv 0(\bmod 2)$. As $\Sigma_{v \in \Sigma}\left|S_{v}(C)\right|=\Sigma_{i \in I}\left|S_{i}(C)\right|$, we also have $\Sigma_{i \in I}\left|S_{i}(C)\right| \equiv 0(\bmod 2)$.

Proof of Theorem 3.1.1. In order to apply Theorem 3.2.1, we have to show that $\mathcal{C}(E, q)$ is connected. Let $C=\left(q_{i}^{v}\right) \in \mathcal{C}(E, q)$, and suppose that there exists $i \in I$ such that $\left|S_{i}(C)\right|$ is odd. By Lemma 3.2.2, we have $\Sigma_{i \in I}\left|S_{i}(C)\right| \equiv 0(\bmod 2)$. Therefore there exists $j \in I$ such that $j \neq i$, and that $\left|S_{j}(C)\right|$ is odd. Since $\Sigma_{i_{0}, i} \neq \Sigma_{k}$ and $\Sigma_{i_{0}, j} \neq \Sigma_{k}$ by hypothesis, $C$ is connected, and hence $\mathcal{C}(E, q)$ is connected. The result now follows from Theorem 3.2.1.

Note that one can give analogs of the results of $\S 3$ in the odd dimensional case. These can be easily deduced from the even dimensional case using the method of [PR, 7.2].

\section{$\S 4$. An example - the case of CM étale algebras}

Recall that a number field is CM if it is a totally imaginary quadratic extension of a totally real number field. Note that a number field is CM if and only if it has exactly one complex conjugation (see for instance [Mi, 1.4.]). We say that $E$ is a CM étale algebra if it is a product of CM number fields, and the complex conjugation of $E$ is by definition the product of the complex conjugations of its factors.

Corollary 4.1.1. Suppose that $E$ is a $C M$ étale algebra of rank $2 n$, and that $\sigma: E \rightarrow E$ is the complex conjugation. Let $q$ be a $2 n$-dimensional quadratic space over $k$. Then $O(q)$ contains a maximal torus of type $(E, \sigma)$ if and only if $\operatorname{disc}(q)=\operatorname{disc}(E) \in k^{*} / k^{* 2}$, the hyperbolicity condition holds and signature of $q$ is even.

Proof. By Lemma 2.2.2, there exists $v \in \Sigma_{k}$ such that for all $i \in I$, we have $v \notin \Sigma_{k}^{\text {split }}\left(K_{i}\right)$, Therefore for all $i, j \in I$ with $i \neq j$, we have $\Sigma_{i, j} \neq \Sigma_{k}$, and we can apply Corollary 3.1.2. As $E$ is $\mathrm{CM}$ and $\sigma$ is the complex conjugation, we have $\rho_{v}=0$, hence the signature condition of Corollary 3.1.2 is equivalent to saying that the signature of $q$ is even.

\section{Bibliography}

[BCM] R. Brusamarello, P. Chuard-Koulmann and J. Morales, Orthogonal groups containing a given maximal torus, J. Algebra 266 (2003), 87-101. 
[F] A. Fiori, Special points on orthogonal symmetric spaces, J. Algebra 372 (2012), 397419.

[GR] S. Garibaldi and A. Rapinchuk, Weakly commensurable S-arithmetic subgroups in almost simple algebraic groups of types B and C, Algebra and Number Theory, to appear.

[G] P. Gille, Type des tores maximaux des groupes semi-simples, J. Ramanujan Math. Soc. 19 (2004), 213-230.

[L] T-Y. Lee, Embedding functors and their arithmetic properties, Comment. Math. Helv, to appear.

[Mi] J. Milne, Complex Multiplication, http://www.jmilne.org/math/CourseNotes/cm.

[M] J. Milnor, Isometries of inner product spaces, Invent. Math. 8 (1969), 83-97.

[O'M] O.T. O'Meara, Introduction to quadratic forms, Reprint of the 1973 edition. Classics in Mathematics. Springer-Verlag, Berlin, 2000.

[PR] G. Prasad and A.S. Rapinchuk, Local-global principles for embedding of fields with involution into simple algebras with involution, Comment. Math. Helv. 85 (2010), 583645 .

[Sch] W. Scharlau, Quadratic and hermitian forms, Grundlehren der Mathematischen Wissenschaften 270, Springer-Verlag, Berlin, 1985.

\author{
Eva Bayer-Fluckiger \\ EPFL-FSB-MATHGEOM-CSAG \\ Station 8 \\ 1015 Lausanne, Switzerland \\ eva.bayer@epfl.ch
}

